\title{
Application of synthetic aperture radar imagery for forward and backward tracking of oil slicks
}

\author{
Tae-Ho Kim ${ }^{1}$, Chan-Su Yang ${ }^{1,2,3, *}$, and Kazuo Ouchi ${ }^{1}$ \\ ${ }^{1}$ Korea Institute of Ocean Science \& Technology, Busan, Republic of Korea \\ ${ }^{2}$ Korea University of Science \& Technology, Daejeon, Republic of Korea \\ ${ }^{3}$ School of Ocean Science and Technology, Busan, Republic of Korea
}

\begin{abstract}
Article history:
Received 31 March 2018

Revised 2 January 2019

Accepted 3 March 2019
\end{abstract}

Keywords:

Oil tracking, Marine weather data, SAR, Oil spill response technique, Backward tracking function

\section{Citation:}

Kim, T.-H., C.-S. Yang, and K. Ouchi, 2019: Application of synthetic aperture radar imagery for forward and backward tracking of oil slicks. Terr. Atmos. Ocean. Sci., 30, 509-519, doi: 10.3319/TAO.2019.03.03.02

\begin{abstract}
This paper presents a technique for tracking of oil slicks movement using synthetic aperture radar (SAR) and external force data with optimal coefficients. The detected oil from the SAR image is used as input data for tracking model, and wind and tidal current data are used as external forces to determine the displacement of oil slick. Forward and backward trackings were performed using a set of 4 SAR images observed at the time of the Hebei Spirit accident in 2007. The movement vectors are calculated using wind and tidal current with various coefficients. All tracking results show more than $56 \%$ accuracy. The new linear equations were calculated using coefficient values with the highest accuracy and velocity values of wind and tidal current. The modified equations were used to back-track from the satellite observation time to the accident occurrence time. The simulation results shows that most of the particles of the spread oil traced back around the point of the accident with little exceptions to some particles which shifted more than the oil spill. This is because the spill time of the particles are different from each other. Thus, the proposed method will contribute to the quick response activities and the estimation of location for the source of oil pollution.
\end{abstract}

\section{INTRODUCTION}

The response technology for oil spill on the ocean can be classified into three types according to the purpose, namely prevention of further spillage, on-site monitoring and risk assessment, and response activity. The monitoring and assessment include the generating geographical information such as the location and movement direction and the quantitative information such as thickness and volume of the spilled oil. The generated information can be efficiently used to carry out the response activity and the strategy modification according to change of the pollution situation (IMO 1988). For this reason, there has been a considerable need to improve the accuracy for the techniques of oil detection (Brekke and Solberg 2005; Solberg et al. 2007; Yang et al. 2013) and movement prediction (Castanedo et al. 2006; Cucco et al. 2012; Zodiatis et al. 2012).

Forward and backward trackings of oil particles in time

\footnotetext{
* Corresponding author

E-mail:yangcs@kiost.ac.kr
}

can provide the future location of oil slicks and the source of an oil spill (Ciappa and Costabile 2014; Kim et al. 2014; Suneel et al. 2016; Tian et al. 2017). This information is very useful for oil pollution response activities. Many systems have been developed to track the trajectory of spilled oil, such as OSCAR (Oil Spill Contingency and Response model), GNOME [General National Oceanic and Atmospheric Administration (NOAA) Operational Modelling Environment], MEDSLIK (3D Oil Spill Model around the Mediterranean, the Black Seas and worldwide), and ADIOS (Automated Data Inquiry for Oil Spills) (Reed et al. 1995; Bergueiro et al. 2006; Zodiatis et al. 2012). However, these models include errors due to local characteristics, i.e., they used fixed values for the coefficient of input marine weather data. Hence, a calibrated model that reflects regional marine and meteorological characteristics is essential.

Numerous studies have been carried out to improve the accuracy of prediction results (Xu et al. 2013; Tian et al. 2017). In particular, the technologies that combine oil through 
satellite imagery and movement models have resulted in significant performance improvements (Yu et al. 2016). Tian et al. (2017) introduced a new method to calibrate Lagrangian model with a set of 4 ENVISAT-ASAR images. They suggest that optimal coefficients of input marine weather data, current drift coefficient of 0.4414 , wind drag coefficient of 0.0338 , wave drift coefficient of 0.5180. Kim et al. (2014) suggested the modified linear equation optimal to estimate optimal wind drift coefficient. These studies, however, did not take into account the spatial changes of tidal current and wind which may cause a large error in coastal waters of shallow waters with varying bottom topography.

In this paper, we have introduced the forward- and backward-tracking simulations to predicted oil trajectories using satellite images. Using a set of 4 synthetic aperture radar (SAR) images, the optimal coefficients for tidal current and wind in each simulation case were suggested. In order to consider the spatial changes new coefficient values were calculated by using the optimal coefficients of each simula- tion case. This method was applied to the Hebei Spirit oil spill accident and quantitatively comparison was carried out with the previously used tracking method.

This paper is organized as follows. In section 2, data are described including the satellite images, wind and tidal current, followed by the description of methodology in section 3 . In section 4, the results are presented, and concluded in section 5 .

\section{DATA}

\subsection{Satellite Data}

In this study, four SAR images acquired by ENVISATASAR, RADARSAT-1, and TerraSAR-X were used for initial position of oil particle and comparison with the simulation results. Figure 1 illustrates the coverage areas, and Table 1 shows the data acquisition time, sensor mode, polarization, swath width, spatial resolution, wind direction, and wind data at the Automatic Weather System (AWS) stations

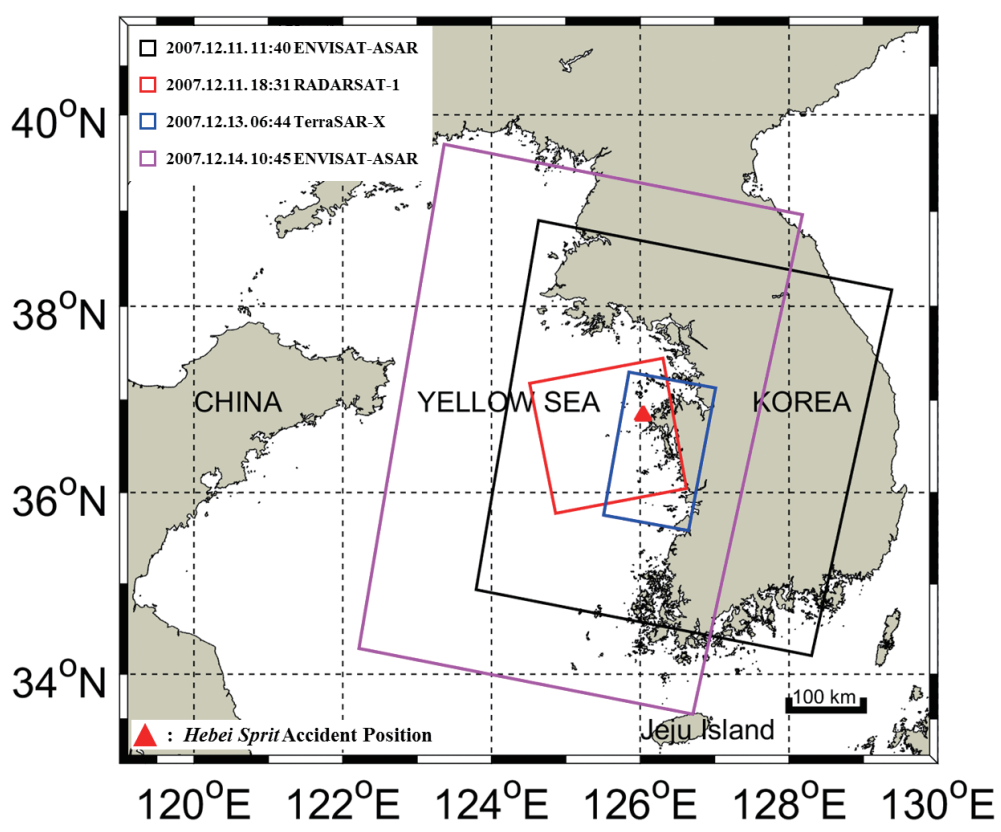

Fig. 1. Coverage of satellite image after the Hebei Spirit oil spill. The boxes represent the boundary of different SAR images as indicated in the legend. The red mark represents location of the oil spill accident.

Table 1. Parameters of satellite sensors and wind data at each acquisition time.

\begin{tabular}{|c|c|c|c|c|}
\hline Satellite & ENVISAT ASAR & RADARSAT-1 & TerraSAR-X & ENVISAT ASAR \\
\hline Acquisition time (LT) & 2007.12.11 10:40 & 2007.12.11 18:31 & 2007.12.13 06:44 & 2007.12.14 10:45 \\
\hline Polarization & VV & $\mathrm{HH}$ & $\mathrm{VV}$ & VV \\
\hline Incidence angle (deg.) & $31.0-36.3$ & $31-39$ & $31.8-40.5$ & $19.2-26.7$ \\
\hline Nominal resolution (m) (range/azimuth) & $150 / 150$ & $25 / 27$ & $18.5 / 18.5$ & $150 / 150$ \\
\hline Wind speed $\left(\mathrm{m} \mathrm{s}^{-1}\right)$ & 5.6 & 6.3 & 4.3 & 6.9 \\
\hline Wind direction (deg.) & 338.0 & 347.0 & 323.1 & 339.0 \\
\hline
\end{tabular}


closest to the centroid of the slick areas at each satellite data acquisition time. For SAR data, the radiometric calibration was first made to calculate normalized radar cross section (NRCS), and geometric correlation was also applied.

\subsection{Current}

The surface current data obtained from Environmental Fluid Dynamics Code (EFDC) (Craig 2012). This model coordinates were generalized by orthogonal curvilinear horizontal and vertical sigma coordinates. It is a 3-dimensional hydrodynamic model based on continuity, momentum, salt balance, and heat balance with hydrostatic and Boussinesq approximations. The model developed at the Virginia Institute of Marine Science (VIMS) and recommended by US Environmental Protection Agency (EPA) for environmental applications. In this study, we used the surface current without wind effect using by the EFDC model with grid spacing of $500 \mathrm{~m}$ (Yang et al. 2009). Surface tidal current data covering the study area were generated at intervals of $30 \mathrm{~min}$. At the oil particle position at a specific time, the velocity data is designed to bring the data of near-real time at $30 \mathrm{~min}$ interval.

\subsection{Wind}

The wind field over the sea area is acquired from the Korea Meteorological Administration (KMA) (https://data. kma.go.kr/cmmn/main.do). The data contain the wind speed and direction averaged over $1 \mathrm{~min}$ at $1 \mathrm{~min}$ intervals at different heights. In order to convert the wind into sea surface wind at a height of $10 \mathrm{~m}$ Hellman exponential law was used. Wind data were obtained from 19 AWS stations around the position of collision. And, we used the interpolation method to generate the wind field data of the study area every $30 \mathrm{~min}$.

\section{METHODOLOGY}

\subsection{Oil Particles from SAR Images}

In this study, we have distinguished the oil and sea pixels around the oil spill area using threshold value. Then, the oil slick areas were determined by analyzing the histograms of NRCS in region of interest and visual inspection on the basis of response reports for the Hebei Spirit oil spill. In order to enter the information of the oil detected from the SAR image into the model, the positions of the detected slick areas were coordinated (Fig. 2). The oil information detected in the SAR images was extracted in the binary image format. In order to generate the oil particles which used as initial data in the model, the image was divided by a certain grid spacing and the position information of the grid contained in the oil area was extracted. In this study, the position information is assumed to be oil particles. Since the size of the oil area extracted from each SAR image is different, a resampling process was performed using the adjusted lattice spacing to unify the number of input oil particles.

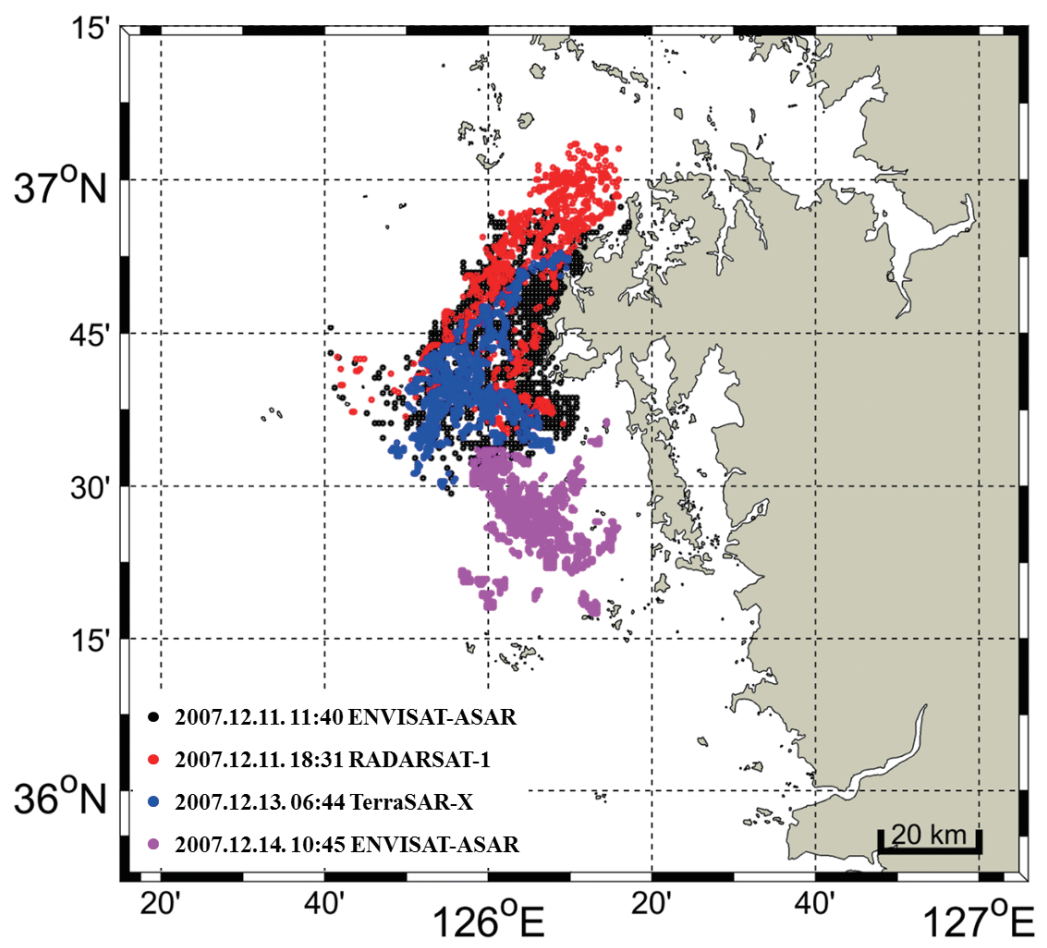

Fig. 2. Oil detection results from SAR images. Black, red, blue, and magenta marks represent oil particles from ENVISAT-ASAR (2007.12.11 10:40 LT), RADASRSAT-1 (2007.12.11 18:31 LT), TerraSAR-X (2007.12.13 06:44 LT), and ENVISAT-ASAR (2007.12.14 10:45 LT) respectively. 
The adjusted lattice spacing was set so that the number of extracted oil particles was close to 900 , and those unified oil particles were calculated using a random sampling method.

\subsection{Tracking Model}

Spilled oil on the ocean surface moves with the current flow and its fate is regulated by various processes such as evaporation, emulsification, natural dispersion, beaching, etc. (Reed et al. 1999). The accurate prediction of the oil movement and fate requires various environmental conditions such as winds, currents, waves, turbulence, salinity, temperature, solar insolation and bottom topography in shallow water, etc. Thus, sophisticated prediction models involve complex computational processes and numerous input data (Reed et al. 1999; Korotenko et al. 2004; Zodiatis et al. 2012). However, some response systems have used models that take into account only the essential elements for quick response (Castanedo et al. 2006; Abascal et al. 2009), and used satellite data to improve the accuracy (Dietrich et al. 2012; Xu et al. 2013; Kim et al. 2014). In this study, a model is designed to use satellite data as initial input data. Also, wind and current velocity with the drift coefficients are applied as external force (Fig. 3). The two-dimensional Lagrangian transport model is used to calculate the trajectories of oil slicks. It takes the particles' positions in the following way: at every step, the movements induced by wind, current and turbulent diffusion are computed for each oil particle, and then the new position of each particle is obtained by super positioning of its movement and current position. The equations are as follows:

$x_{i}^{t+\Delta t}=x_{i}^{t}+v_{i}^{t} \Delta t+u_{d}$
$x_{i}^{t-\Delta t}=x_{i}^{t}-v_{i}^{t} \Delta t+u_{d}$

where Eqs. (1) and (2) represent forward and backward tracking methods, respectively, and $x_{i}^{t}$ and $x_{i}^{t \pm \Delta t}$ are location of the $i^{\text {th }}$ particle at the times of $t$ and $t \pm \Delta t$, respectively; and $v_{i}^{t}$ is the velocity of the $i^{\text {th }}$ particle at the time of $t$, calculated as the linear combination of the wind and current velocities, expressed as:

$v_{i}^{t}=C_{c} C_{i}^{t}+C_{w} W_{i}^{t}$

where $C_{i}^{t}$ and $W_{i}^{t}$ are the surface current velocity and wind velocity, respectively, where the subscript $i$ and superscript $t$ indicate the particles' location and time, respectively. The wind velocity is at $10 \mathrm{~m}$ height over the sea surface. $u_{d}$ is the turbulent diffusive velocity:

$u_{d}=(2 R-1) \sqrt{ }(c E v \Delta t)$

where $R$ is a random number in the interval between 0 and $1, E v\left(10 \mathrm{~m}^{2} \mathrm{~s}^{-1}\right)$ is the turbulent coefficient, and $c(12)$ is a scaling coefficient (Proctor et al. 1994; Dietrich et al. 2012).

\subsection{Tracking Simulation}

The process of oil slick tracking involves both advective and diffusive velocities. The diffusion velocity is dominated by random walk, and weathering processes including evaporative and decaying processes as well as diffusive velocity have not been incorporated into this model. The forwardand backward-tracking simulations were carried out for 3

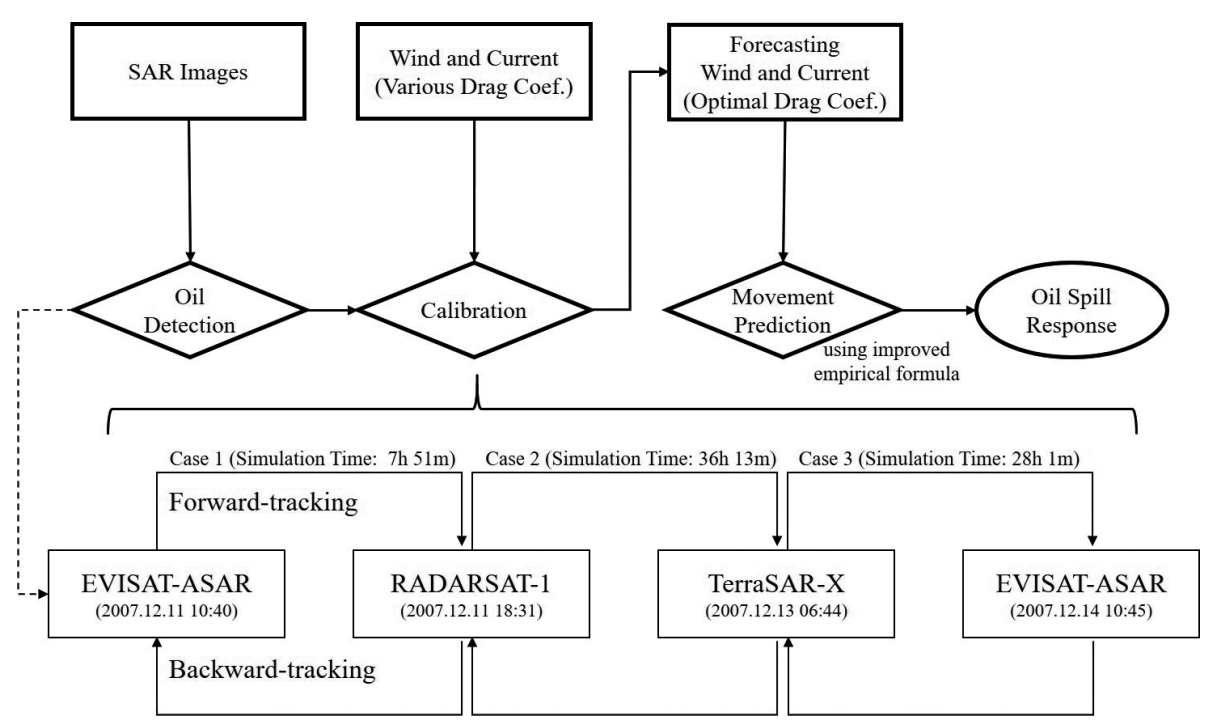

Fig. 3. Design of forward- and backward-tracking models using satellite images. In the calibration step, simulation were carried out for a total of 6 cases to estimate optimal drag coefficients. 
cases. In the case 1 of the forward-tracking model, the oil particles from EVISAT-ASAR image were inputted into the simulation as initial data for generation of trajectories at the acquisition time of RADARSAT-1 image. In the same way, the RADARSAT- 1 and TerraSAR-X images were used as the initial data for the cases 2 and 3 respectively. The backward simulation is in the reversed direction in time to the forward simulation. For example, in the case 1 the image of RADARSAT-1 is input and simulated up to the observation time of ENVISAT-ASAR. The simulation time of each case was set as the difference of observation time of satellite images. The case 1 had the shortest difference time of $7 \mathrm{~h}$ and $51 \mathrm{~min}$ between the start and end time, and the case 2 had the longest time of $36 \mathrm{~h}$ and $13 \mathrm{~min}$ (Table 2). To get an optimal combination of two coefficients in Eq. (3), namely $C_{c}, C_{w}$, quantitative evaluation of simulation was carried out using various marine environmental information. The tidal current drift coefficient was applied in the range of $100-120 \%$ at $1 \%$ interval. Also, the movement simulation of oil particles was simulated using wind drift coefficient with values ranging from $1-5 \%$ at intervals of $0.1 \%$. In order to calculate the optimum coefficient value, a total of 1020 simulation results and the satellite data were compared quantitatively. Quantitative evaluation was done by lattice cells method (Kim et al. 2014). In the present study, the same cell size $(500 \times 500 \mathrm{~m})$ as the current and wind fields were used. The results of the calculation of optimal coefficients are described in details in section 4 .

\section{RESULTS}

\subsection{Estimation of Optimal Coefficient}

The accuracy of the simulation results is compared with the satellite observation information. Figure 4 shows the forward- and backward-tracking comparison results which have highest matching rate of over $56 \%$. Table 3 shows the values of the highest matching ratio among the results of the simulation and the values of the tidal current and wind coefficients applied to the simulation. In this study, only two-dimensional wind and current information were used as external forces; thus, factors like weathering effect and bottom topography were not considered. Indeed, in coastal waters the external force due to waves can be an important factor in determining the movement of oil particles. Another important reason for determining accuracy is the temporal and spatial variation of marine weather data. Figures 5 and 6 show time series data of the wind and tidal current at the midpoint of the detected oil area from each satellite. Tidal current shows repetitive changes in the north-south direction, and the velocity shows almost the same value (Fig. 6). However, it shows a somewhat weaker speed in the last detected oil area (Fig. 6d). Wind variations, on the other hand, vary greatly with time and space (Fig. 5). In case 2, relatively weak winds were observed, while in case 3 , strong winds persisted in the south direction. In addition, the last detected oil area showed a very different wind change pattern from the other areas (Fig. 5d). Therefore, the reason for the relatively low accuracy of case 3 is that it uses a fixed coefficient value without considering the change of the temporal and spatial marine weather data.

In order to perform the simulation considering the temporal and spatial changes of the marine weather data new coefficient values were calculated using the coefficients applied when the highest matching results were obtained.

$v_{i}{ }^{t}=C_{c}{ }^{\prime} C_{i}^{t}+C_{w}{ }^{\prime} W_{i}^{t}$

where $v_{i}^{{ }_{t}}$ is the advection velocity of $i^{\text {th }}$ oil particle at the time of $t . C_{c}{ }^{\prime}$ and $C_{w}{ }^{\prime}$ are the calculated current drift and wind drag coefficients, respectively:

$C_{c}{ }^{\prime}=a C_{i}^{t}+b$

$C_{w}{ }^{\prime}=c W_{i}^{t}-d$

The coefficients $a, b, c$, and $d$ are given by $46.699 \times 10^{-2}$ $\left(\mathrm{s} \mathrm{m}^{-1}\right), 75.523 \times 10^{-2}, 0.911 \times 10^{-2}\left(\mathrm{~s} \mathrm{~m}^{-1}\right)$, and $1.518 \times 10^{-2}$, respectively (Fig. 7). This formula can reflect the time and spatial changes of wind speed and tidal current. However, when used in other areas, it must be checked for suitability.

\subsection{Backward-tracking of the Hebei Spirit Accident}

The purpose of backtracking is to locate the source of the pollutants or resource exploration. In this study, we evaluated the possibility of backward-tracking in time to the location of the Hebei Spirit accident in 2007 using only

Table 2. Start and end times for forward and backward tracking simulation and time difference for cases $1-3$.

\begin{tabular}{|c|c|c|c|}
\hline & $\begin{array}{l}\text { Forward: Start time (LT) } \\
\text { Backward: End time (LT) }\end{array}$ & $\begin{array}{l}\text { Forward: End time (LT) } \\
\text { Backward: Start time (LT) }\end{array}$ & Time difference \\
\hline Case 1 & $12.11 .10: 40$ & $12.11 .18: 31$ & 7 h 51 min \\
\hline Case 2 & $12.11 .18: 31$ & 12.13. 06:43 & $36 \mathrm{~h} 13 \mathrm{~min}$ \\
\hline Case 3 & $12.13 .06: 43$ & 12.14. 10:45 & $28 \mathrm{~h} 01 \mathrm{~min}$ \\
\hline
\end{tabular}



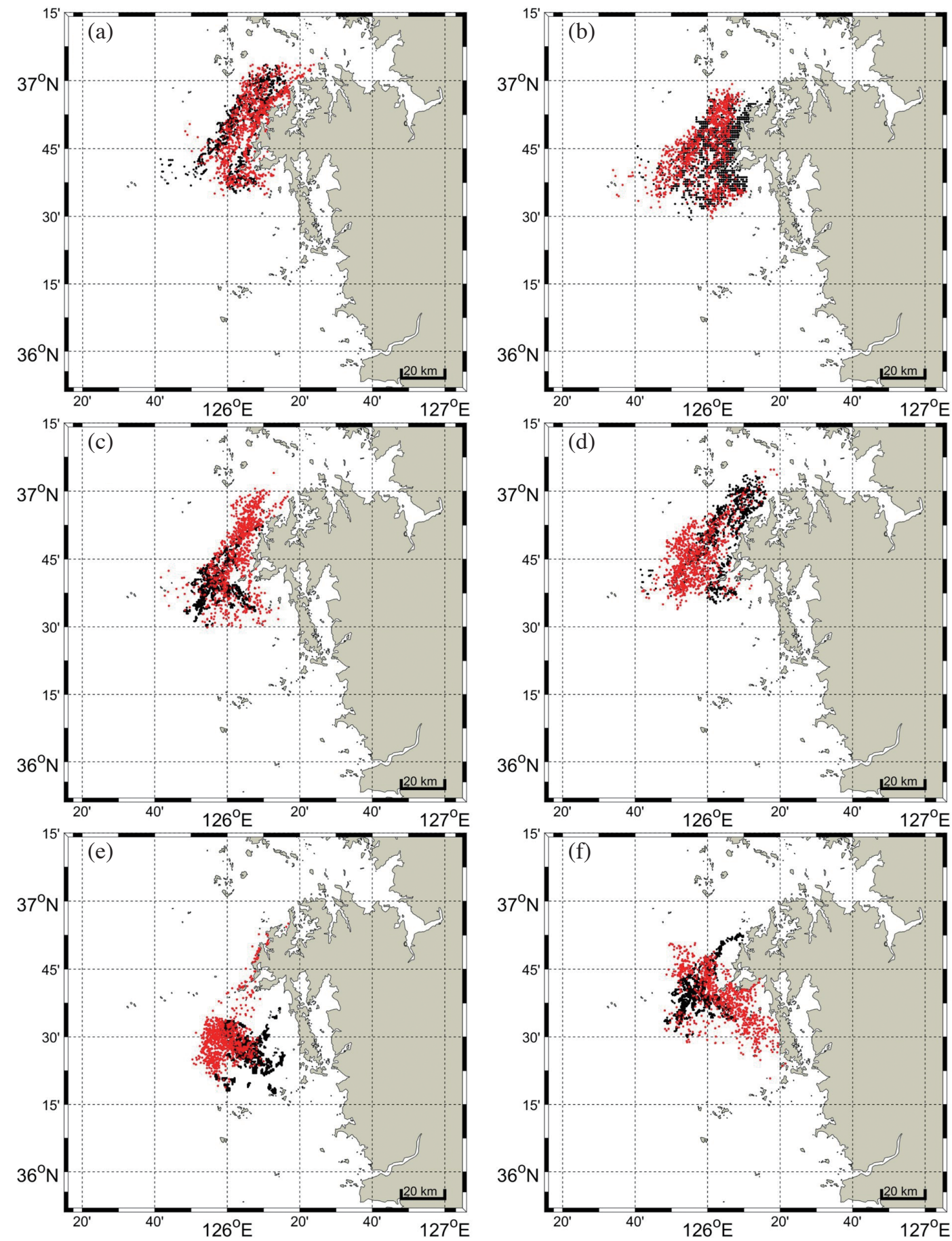

Fig. 4. Comparison between observed and forward- and backward-simulated oil slicks for case 1: (a) and (b), case 2: (c) and (d), and case 3: (e) and (f), respectively. Left and right column represent forward- and backward-tracking results. The black and red marks represent observed and simulated oil slicks, respectively. The black marks in (b) extracted from ENVISAT-ASAR (2007.12.11 10:40 LT). The observed oil slicks in (a), (d), and (c), (f) detected from RADARSAT-1 (2007.12.11 18:31 LT) and TerraSAR-X (2007.12.13 06:44 LT), respectively. The black marks in (e) represent observed oil slicks from ENVISAT-ASAR (2007.12.14 10:45 LT). 
Table 3. Highest matching rate between simulation result and satellite data, and the drag coefficients of tidal current and wind.

\begin{tabular}{l|lc|cc}
\hline & \multicolumn{2}{|c|}{ Forward } & \multicolumn{2}{c}{ Backward } \\
\cline { 2 - 5 } & Condition (\%) & Matching Rate & Condition $(\%)$ & Matching Rate \\
\hline Case 1 & $C_{w}: 1.0, C_{c}: 114$ & 69.32 & $C_{w}: 2.0, C_{c}: 101$ & 56.22 \\
Case 2 & $C_{w}: 1.3, C_{c}: 103$ & 91.08 & $C_{w}: 1.3, C_{c}: 113$ & 76.76 \\
Case 3 & $C_{w}: 3.1, C_{c}: 110$ & 69.10 & $C_{w}: 2.9, C_{c}: 100$ & 72.59 \\
\hline
\end{tabular}

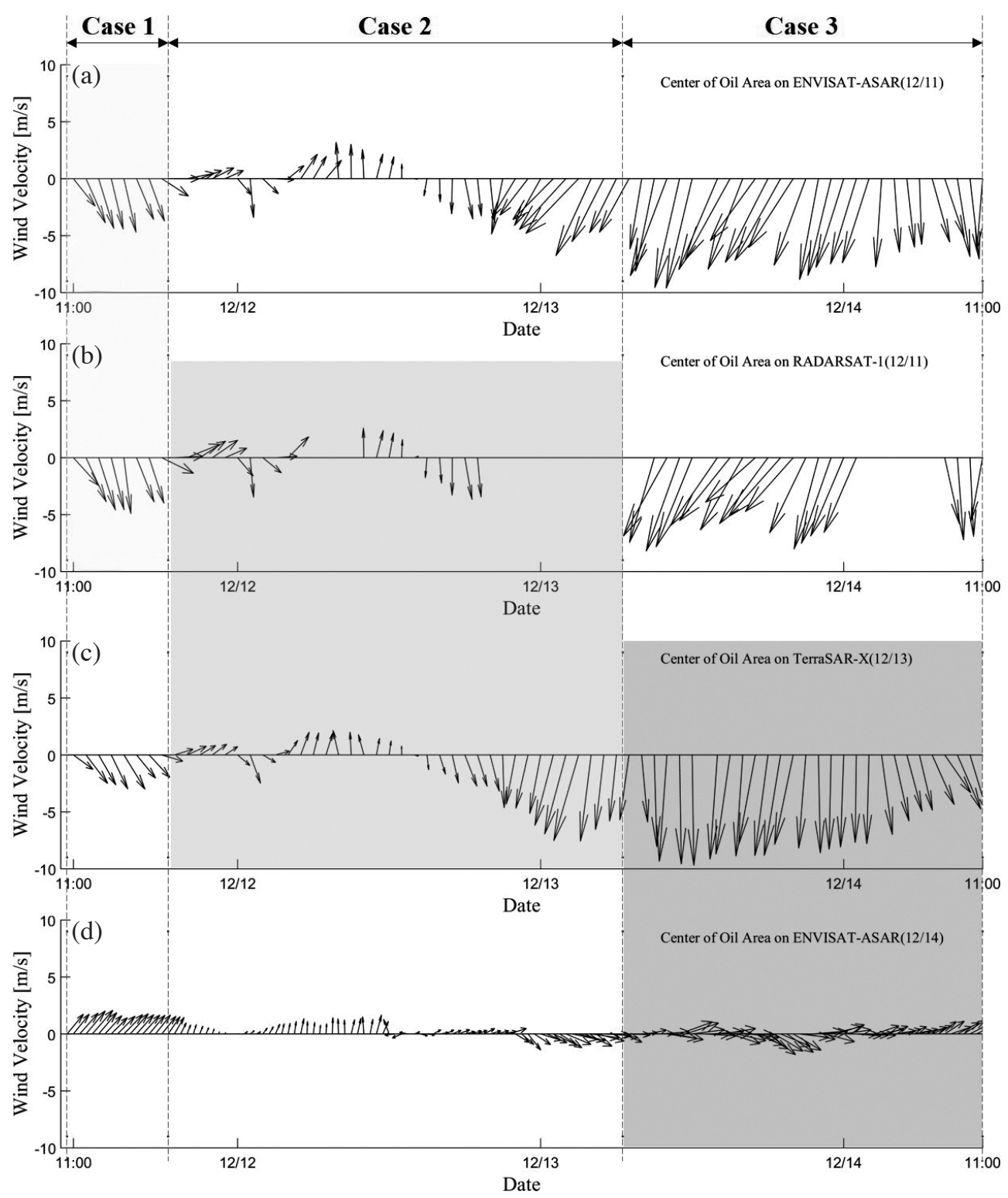

Fig. 5. Wind vector at the center of detected oil area from each SAR image during simulation time. The light grey, grey, and dark grey boxes represent the duration of case 1,2 and 3, respectively. 


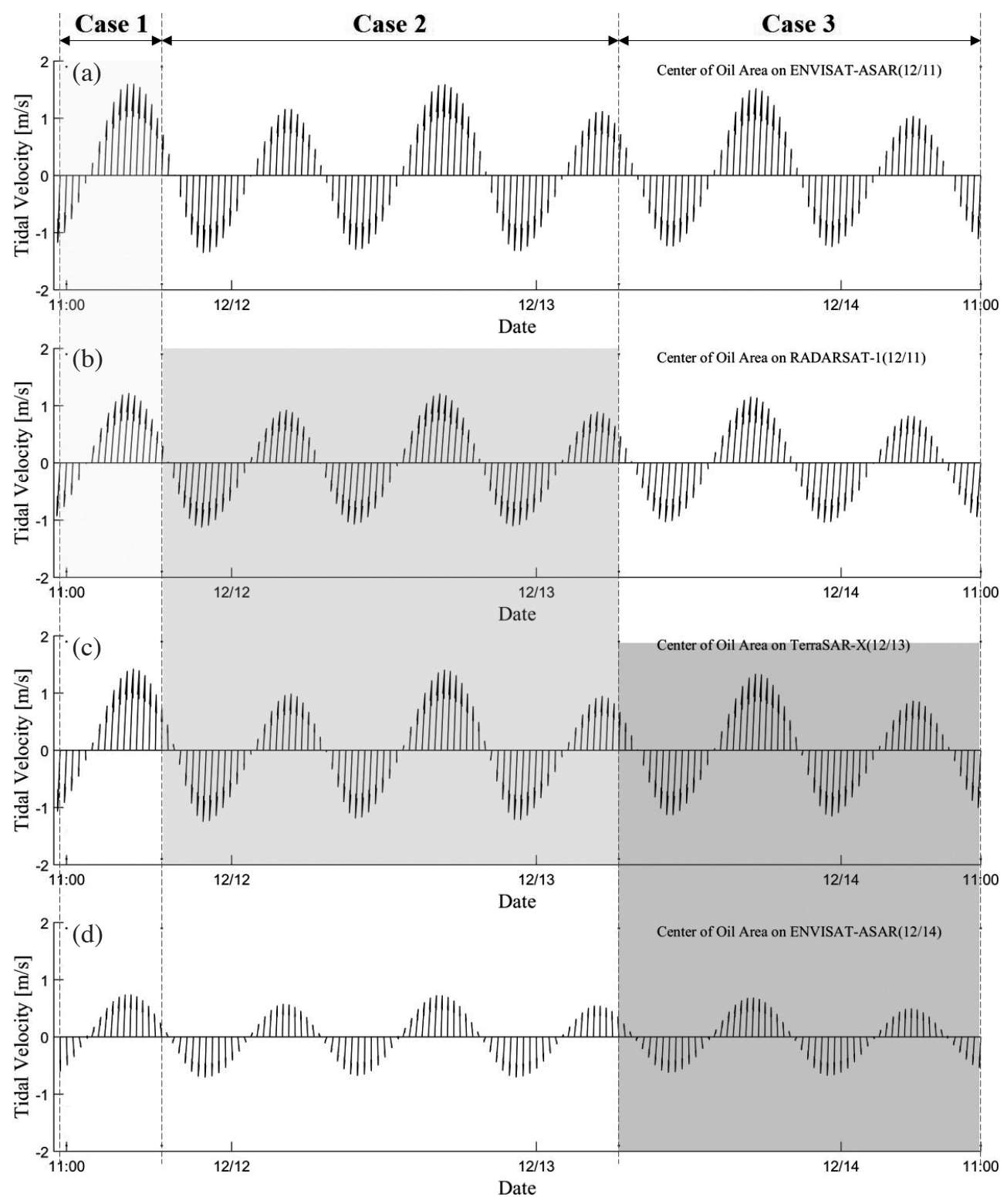

Fig. 6. Tidal current vector at the center of detected oil area from each SAR image during simulation time. The light grey, grey, and dark grey boxes represent the duration of case 1,2 and 3 , respectively.
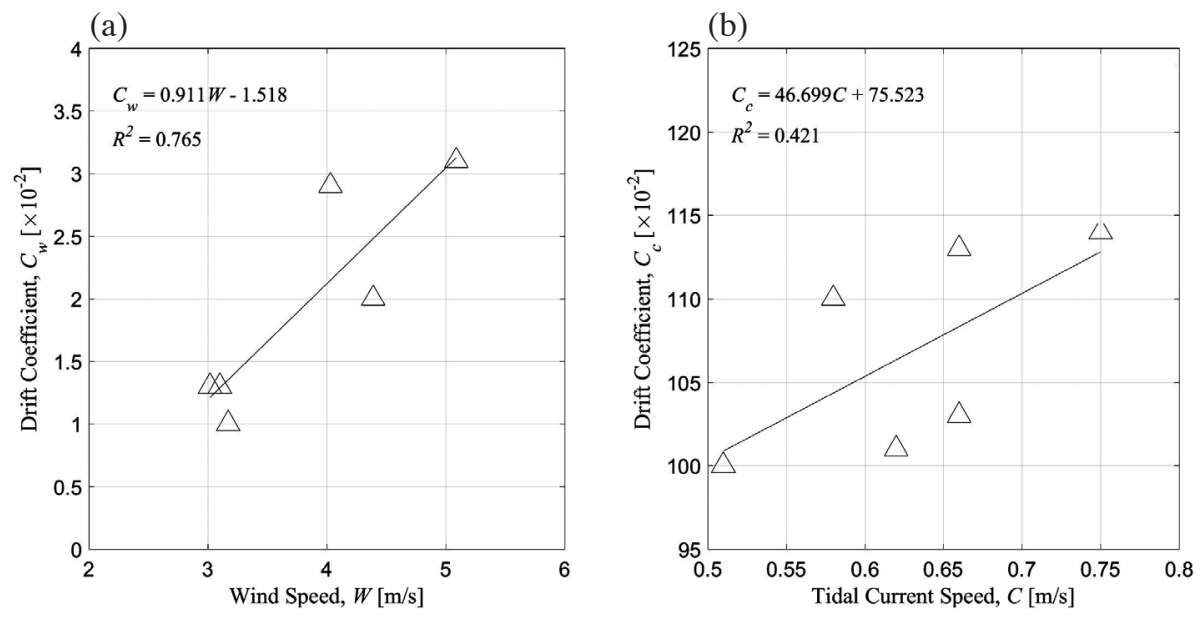

Fig. 7. Scatter diagram of the drift coefficients and speed. (a) and (b) represent wind speed and tidal current speed, respectively. 
wind and tidal current data with improved linear equations. The KOMPSAT-2 image was used as the input satellite data for its observation time was closest to the time of the accident among other satellite data (Yang et al. 2009). KOMPSAT-2 can acquire panchromatic image of $1 \mathrm{~m}$ resolution and four bands multispectral images of $4 \mathrm{~m}$ resolution. In this study, we used the multispectral image to extract oil spilled area. As the oil has continuously spilled out from the accident time to the satellite observation time, it is not appropriate to use the entire oil detection result as input data for the backward-tracking model. Therefore, it is necessary to perform backtracking by separating the oil from the satellite image at the time similar to the time when the accident occurred. In order to distinguish the spilled oil at the beginning of an accident based on the position of the detected oil in the satellite image, one oil particle was forward-tracked from the accident location to the KOMPSAT-2 observation time. The simulation result shows that spilled oil at the time of the accident moved from the location of the Hebei Spirit accident to the south-eastern coast. Therefore, the detected oil particles located in this area were assumed to be oil spilled at the time of the accident. The oil particles in the grey box in Fig. 8 were entered as initial position of oil particles and backward tracking was performed at the time of the accident (Fig. 9). It was confirmed that the oil particles move close to the point of accident with time while showing the characteristic of repetitive motion by strong tidal current. The distance error of the backward tracking result was quantitatively evaluated using the positions of backtracked oil particles corresponding to the accident time within $3 \mathrm{~h}$. From the distance between the 765 oil particle positions and the accident location, it is found that the minimum error is $0.08 \mathrm{~km}$, and the maximum error is $16.14 \mathrm{~km}$ with an average error of $6.43 \mathrm{~km}$. The results of this model show that the use of the coefficient correction formula and satellites can predict the direction of oil particle movement and the oil position including errors within several $\mathrm{km}$ even if only wind and current are used. Therefore, when satellite and correction formula are used, it is possible to obtain information useful for establishing an initial response plan for an oil spill accident using minimum information. For more accurate error analysis, it may be necessary to use a nonlinear equation-based simulation model that takes account of the acceleration of oil particles during backtracking. In addition, a simulation is required that takes the actual emission time of the input particles of the simulation into account.

\section{CONCLUSIONS}

This paper presents a technique for fast tracking of oil slicks movement using SAR and marine weather data with optimal coefficients. Forward- and backward-trackings were performed using 4 SAR images observed at the time of the Hebei Spirit accident in 2017. All tracking results show more than $56 \%$ accuracy. The new linear equations were calculated using the highest accuracy calculated coefficient values, and wind and tidal current velocity values. The modified equations were used for back-tracking from

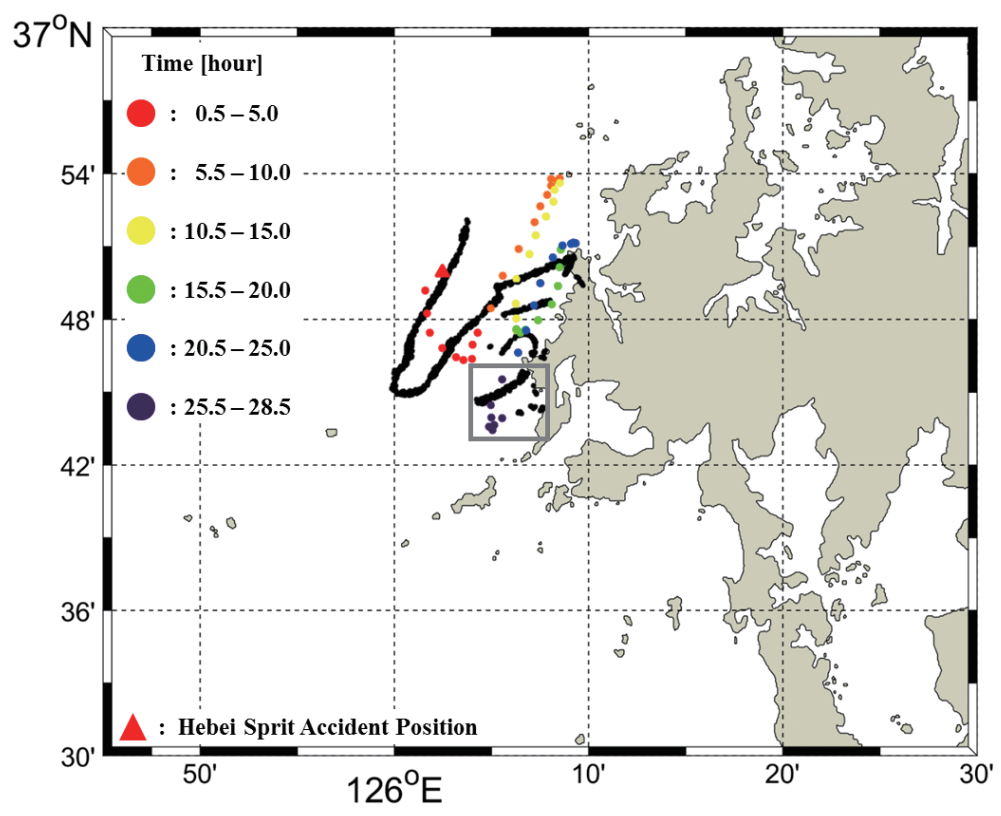

Fig. 8. The black marks indicate oil detection result from KOMPSAT-2 image (2007.12.08 11:04 LT). The other color markers indicate the predicted oil positions every $30 \mathrm{~min}$ from the time of the accident to the observation of the satellite. From red to blue color markers, they show the predicted oil location for every $5 \mathrm{~h}$ from the accident to $25 \mathrm{~h}$ after the accident. Purple marks represent the predicted oil position from $25.5 \mathrm{~h}$ to the time of satellite observation. The grey box is used to extract the initial oil particles for backward-tracking simulation. The red triangle represents the accident position of the Hebei Spirit. 


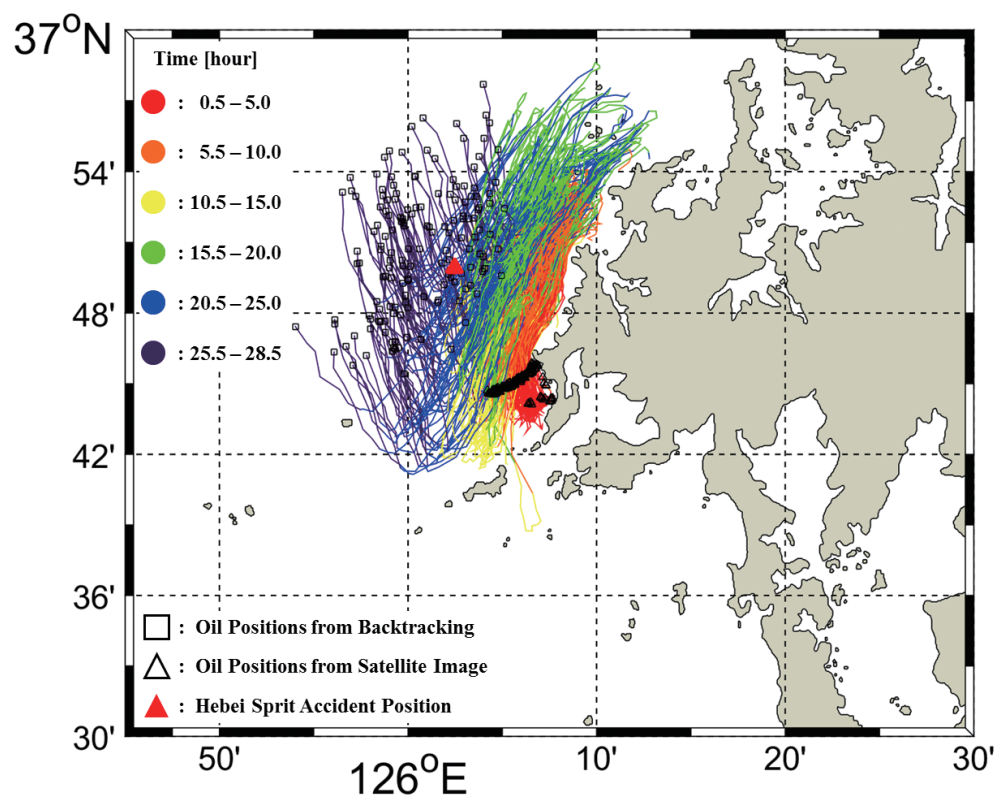

Fig. 9. The black triangle markers represent initial oil particle for backward-tracking simulation. The color lines indicate trajectories from the time of the satellite observation to the Hebei Spirit accident. The black square marks show end position of oil particles at the time of the accident. The red triangle represents the accident position of the Hebei Spirit oil spill.

the satellite observation time to the time of the accident. The simulation results showed that the particles of oil were backtracked around the point of the accident. However, some particles were found to move more than the oil spill by this back-tracking. This is because the spill time of the particles are different for different particles. The cause of this result should be studied using additional data and more precise simulations. This result will contribute to increase the efficiency of practical application for maritime spill response and regulation of illegal oil spill activities. Especially, it is expected to be used for the source point estimation and resource exploration using the backward-tracking function.

Acknowledgements This study is done under the support of the projects "Development of Ship-handling and Passenger Evacuation Support System", and "Construction of Ocean Research Stations \& their Application Studies" funded by the Ministry of Oceans and Fisheries, Korea, and "Development of Technology on Survivor Search and Survivability Assurance in the Accident Ship" funded by the Korea Coast Guard, Korea.

\section{REFERENCES}

Abascal, A. J., S. Castanedo, F. J. Mendez, R. Medina, and I. J. Losada, 2009: Calibration of a Lagrangian transport model using drifting buoys deployed during the Prestige Oil Spill. J. Coast. Res., 251, 80-90, doi: 10.2112/07-0849.1. [Link]

Bergueiro, J. R., R. R. March, S. G. González, and F. S.
Socías, 2006: Simulation of oil spills at the Casablanca platform (Tarragona, Spain) under different environmental conditions. J. Marit. Res., 3, 55-72.

Brekke, C. and A. H. S. Solberg, 2005: Oil spill detection by satellite remote sensing. Remote Sens. Environ., 95, 1-13, doi: 10.1016/j.rse.2004.11.015. [Link]

Castanedo, S., R. Medina, I. J. Losada, C. Vidal, F. J. Méndez, A. Osorio, J. A. Juanes, and A. Puente, 2006: The prestige oil spill in Cantabria (Bay of Biscay). Part I: Operational forecasting system for quick response, risk assessment, and protection of natural resources. $J$. Coast. Res., 226, 1474-1489, doi: 10.2112/04-0364.1. [Link]

Ciappa, A. and S. Costabile, 2014: Oil spill hazard assessment using a reverse trajectory method for the Egadi marine protected area (Central Mediterranean Sea). Mar. Pollut. Bull., 84, 44-55, doi: 10.1016/j.marpolbul.2014.05.044. [Link]

Craig, P., 2012: User's Manual for EFDC_Explorer: A Pre/ Post Processor tor the Environmental Fluid Dynamics Code, Knoxville: Dynamic Solutions-International, LLC.

Cucco, A., M. Sinerchia, A. Ribotti, A. Olita, L. Fazioli, A. Perilli, B. Sorgente, M. Borghini, K. Schroeder, and R. Sorgente, 2012: A high-resolution real-time forecasting system for predicting the fate of oil spills in the Strait of Bonifacio (western Mediterranean Sea). Mar. Pollut. Bull., 64, 1186-1200, doi: 10.1016/j.marpolbul.2012.03.019. [Link]

Dietrich, J. C., C. J. Trahan, M. T. Howard, J. G. Fleming, 
R. J. Weaver, S. Tanaka, L. Yu, R. A. Luettich, C. N. Dawson, J. J. Westerink, G. Wells, A. Lu, K. Vega, A. Kubach, K. M. Dresback, R. L. Kolar, C. Kaiser, and R. R. Twilley, 2012: Surface trajectories of oil transport along the Northern Coastline of the Gulf of Mexico. Cont. Shelf Res., 41, 17-47, doi: 10.1016/j. csr.2012.03.015. [Link]

IMO, 1988: Manual on Oil Pollution, Section IV, International Maritime Organization (IMO), London, England, $216 \mathrm{pp}$.

Kim, T. H., C. S. Yang, J. H. Oh, and K. Ouchi, 2014: Analysis of the contribution of wind drift factor to oil slick movement under strong tidal condition: Hebei Spirit oil spill case. PLOS ONE, 9, e87393, doi: 10.1371/ journal.pone.0087393. [Link]

Korotenko, K. A., R. M. Mamedov, A. E. Kontar, and L. A. Korotenko, 2004: Particle tracking method in the approach for prediction of oil slick transport in the sea: Modelling oil pollution resulting from river input. J. Mar. Syst., 48, 159-170, doi: 10.1016/j.jmarsys.2003.11.023. [Link]

Proctor, R., R. A. Flather, and A. J. Elliott, 1994: Modelling tides and surface drift in the Arabian Gulf-application to the Gulf oil spill. Cont. Shelf Res., 14, 531-545, doi: 10.1016/0278-4343(94)90102-3. [Link]

Reed, M., O. M. Aamo, and P. S. Daling, 1995: Quantitative analysis of alternate oil spill response strategies using OSCAR. Spill Science \& Technology Bulletin, 2, 6774, doi: 10.1016/1353-2561(95)00020-5. [Link]

Reed, M., Ø. Johansen, P. J. Brandvik, P. Daling, A. Lewis, R. Fiocco, D. Mackay, and R. Prentki, 1999: Oil spill modeling towards the close of the $20^{\text {th }}$ century: Overview of the state of the art. Spill Science \& Technology Bulletin, 5, 3-16, doi: 10.1016/S1353-2561(98)000292. [Link]
Solberg, A. H. S., C. Brekke, and P. O. Husøy, 2007: Oil Spill Detection in Radarsat and Envisat SAR Images. IEEE Trans. Geosci. Remote Sensing, 45, 746-755, doi: 10.1109/TGRS.2006.887019. [Link]

Suneel, V., A. Ciappa, and P. Vethamony, 2016: Backtrack modeling to locate the origin of tar balls depositing along the west coast of India. Sci. Total Environ., 569-570, 31-39, doi: 10.1016/j.scitotenv.2016.06.101. [Link]

Tian, S., X. Huang, and H. Li, 2017: A new method to calibrate Lagrangian model with ASAR images for oil slick trajectory. Mar. Pollut. Bull., 116, 95-102, doi: 10.1016/j.marpolbul.2016.12.054. [Link]

Xu, Q., X. Li, Y. Wei, Z. Tang, Y. Cheng, and W. G. Pichel, 2013: Satellite observations and modeling of oil spill trajectories in the Bohai Sea. Mar. Pollut. Bull., 71, 107-116, doi: 10.1016/j.marpolbul.2013.03.028. [Link]

Yang, C. S., D. Y. Kim, and J. H. Oh, 2009: Study on improvement of oil spill prediction using satellite data and oil-spill model: Hebei Spirit Oil Spill. Kor. J. Rem. Sens., 25, 435-444. (in Korean)

Yang, C. S., S. M. Park, Y. Oh, and K. Ouchi, 2013: An analysis of the radar backscatter from oil-covered sea surfaces using moment method and Monte-Carlo simulation: Preliminary results. Acta Oceanol. Sin., 32, 5967, doi: 10.1007/s13131-013-0267-7. [Link]

Yu, F., J. Li, S. Cui, Y. Zhao, Q. Feng, and G. Chen, 2016: A hindcast method to simulate oil spill trajectories for the Bohai Sea, Northeast China. Ocean Eng., 124, 363370, doi: 10.1016/j.oceaneng.2016.08.004. [Link]

Zodiatis, G., R. Lardner, D. Solovyov, X. Panayidou, and M. De Dominicis, 2012: Predictions for oil slicks detected from satellite images using MyOcean forecasting data. Ocean Sci., 8, 1105-1115, doi: 10.5194/os-81105-2012. [Link] 